\title{
The Interesting Influence of Nanosprings on the Viscoelasticity of Elastomeric Polymer Materials: Simulation and Experiment
}

\author{
Jun Liu, Yong-Lai Lu, Ming Tian, Fen Li, Jianxiang Shen, Yangyang Gao, \\ and Liqun Zhang*
}

Among all carbon nanostructured materials, helical nanosprings or nanocoils have attracted particular interest as a result of their special mechanical behavior. Here, carbon nanosprings are used to adjust the viscoelasticity and reduce the resulting hysteresis loss $(\mathrm{HL})$ of elastomeric polymer materials. Two types of nanospring-filled elastomer composites are constructed as follows: system I is obtained by directly blending polymer chains with nanosprings; system II is composed of the self-assembly of a tri-block structure such as chain-nanospring-chain. Coarse-grained molecular dynamics simulations show that the incorporation of nanosprings can improve the mechanical strength of the elastomer matrix through nanoreinforcement and considerably decrease the hysteresis loss. This finding is significant for reducing fuel consumption and improving fuel efficiency in the automobile tire industry. Furthermore, it is revealed that the spring constant of nanosprings and the interfacial chemical coupling between chains and nanosprings both play crucial roles in adjusting the viscoelasticity of elastomers. It is inferred that elastomer/carbon nanostructured materials with good flexibility and reversible mechanical response (carbon nanosprings, nanocoils, nanorings, and thin graphene sheets) have both excellent mechanical and low HL properties; this may open a new avenue for fabrication of high performance automobile tires and facilitate the large-scale industrial application of these materials. proposed for CNTs, including energy conservation and storage, ${ }^{[1]}$ gas and optical sensors, ${ }^{[2,3]}$ field-emission displays, ${ }^{[4]}$ nanometer-sized semiconductor devices, ${ }^{[5]}$ high-strength polymer-based CNTs nanocomposites, ${ }^{[6-9]}$ and so on. Amongst all carbon nanostructured materials with different nanoscale morphologies, helical multiwalled carbon nanotubes (MWCNTs) or carbon nanosprings have special mechanical properties for potential technological and engineering applications. For instance, Poggi et al. ${ }^{[10]}$ adopted an atomic force microscope (AFM) to study the compressive response of a single carbon nanospring and da Fonseca et al. ${ }^{[11,12]}$ used the Kirchhoff rod model to analyze the elastic properties of the helical nanosprings. Meanwhile, the tensile behavior of a carbon nanocoil or nanospring was also examined by clamping it between two AFM cantilevers, which showed that the carbon nanocoil behaves like an elastic spring with a spring constant $K$ equal to $0.12 \mathrm{~N} / \mathrm{m}$ in the low strain region. ${ }^{[13]}$ Furthermore, the reversible elastic deformation of carbon nanotube rings through

\section{Introduction}

It is well known that carbon nanotubes (CNTs) have attracted considerable interest attributed to their unique mechanical and electrical properties, and many potential applications have been

Dr. J. Liu, ${ }^{[+]}$Prof. Y.-L. Lu, Prof. M. Tian, F. Li, J. Shen, Y. Gao, Prof. L.-Q. Zhang

Key Laboratory of Beijing City on Preparation and Processing of Novel Polymer Materials Beijing University of Chemical Technology E-mail: zhanglq@mail.buct.edu.cn

Prof. Y.-L. Lu, Prof. M. Tian, Prof. L.-Q. Zhang

State Key Laboratory of Organic-Inorganic Composites

Beijing University of Chemical Technology

Beijing 100029, P. R. China

[+] Present address: Department of Chemical Engineering, University of Michigan, Ann Arbor, MI 48109, USA

DOI: $10.1002 / \mathrm{adfm} .201201438$

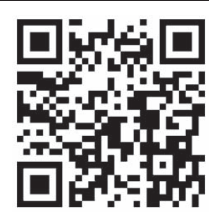

the polymer shells in a colloidal solution was reported. ${ }^{[14]}$ For the single-layer graphene sheet (SLGS), it was as observed that SLGS possesses high flexibility and bends easily in the elastic regime. ${ }^{[15,16]}$ In fact, recently Xu et al. ${ }^{[17-19]}$ prepared a viscoelastic material composed from a random network of long interconnected carbon nanotubes, which exhibited an operational temperature ranging from -196 degrees to 1000 degrees. This underlying mechanism results from energy dissipation through the zipping and unzipping of carbon nanotubes at contacts. Their research work was later highlighted by Gogotsi ${ }^{[20]}$ and $\mathrm{Dai},{ }^{[21]}$ emphasizing the breakthrough of the preparation of the high-temperature elastomeric viscoelastic materials from carbon nanotubes.

According to these examples, it can be seen that the mechanical behavior of these carbon nanostructured materials has received particular interest and they always exhibit good elasticity and flexibility. In fact, although CNTs have extraordinary mechanical strength with an elastic modulus on the order of 
$1 \mathrm{TPa}$ and a shear modulus of approximately $1 \mathrm{GPa}$, single tube is easy to bend and buckle. For example, through high resolution electron microscope and atomistic simulations, Iijima et al. ${ }^{[22]}$ have shown that the bending of the CNTs is fully reversible up to very large bending angles, despite the occurrence of kinks and highly strained tube regions. Meanwhile, using AFM, Falvo et al. ${ }^{[23]}$ have observed that MWCNTs can be bent repeatedly through large angles without undergoing breakage.

In this work, our main aim is to further exploit the technological application of these carbon nanostructured materials by using carbon nanosprings to adjust the viscoelasticity and hysteresis loss (HL) of elastomeric polymer materials during tension-recovery process. This new idea is based on the following two considerations: the first is that the introduction of nanosprings into elastomeric matrix could significantly improve its mechanical properties, such as Young's modulus and tensile strength through nanoreinforcement. ${ }^{[24,25]}$ The second is that in the case of strong interfacial interaction between macromolecular chains and nanosprings, and good dispersion of nanosprings, the nanosprings will store and release energy during the loading-unloading period, and this reversible deformation and recovery of nanosprings may also bring the surrounding macromolecular chains to move together, which is believed to reduce the internal friction between macromolecular chains and decrease the resulting hysteresis loss (HL).

In fact, hysteresis loss (HL) is one important parameter of the viscoelasticities of elastomeric polymers. ${ }^{[26]}$ Although the mechanical properties of elastomers are greatly enhanced by traditional nanofillers such as carbon black and silica, the HL increases significantly attributed to the formation of the three-dimensional network of nanofillers, and its destructionreconstruction under periodical stress leads to much more internal frictions between filler-filler, filler-molecular chains and molecular chains-molecular chains, compared with unfilled elastomer. ${ }^{[27]}$ Moreover, HL is the main origin of rolling resistance of rubber tires, which undoubtedly is extremely disadvantageous for saving fuel consumption and improving fuel efficiency in the tire industry. In the meantime, the heat generation and accumulation inside rubber tires caused by dynamic heteresis loss will inevitably decrease the tire materials' strength and may cause the tire to be broken much earlier than its normal service life. It is estimated that $40 \%$ of the total fuel consumption of an automibile results from the rolling resistance of automobile tires ${ }^{[28]}$ which is caused mainly by dynamic hysteresis, namely, the energy used to deform the rubber will be partially converted to heat. Taking into account the more and more strict energy crisis facing the world today, and such a large amount of the usage of automobile tires, how to effectively decrease the HL even by several percentages will be a great contribution and breakthrough.

Hence, the proposed idea by using the nanosprings to adjust the viscoelasticity and reduce the HL of elastomeric polymer materials is of great significance, which is originally enlightened and motivated by the excellent recoverable mechanical response of nanosprings. Evidently, our work is completely contrary to the interesting work done by $\mathrm{Xu}$ et al., ${ }^{[17-19]}$ who aimed to increase the viscosity and the damping ratio or HL of carbon nanotube material through a zipping and unzipping of carbon nanotubes at contacts. Here we mainly adopt coarse-grained molecular dynamics simulation (CGMDS) and our simulated results indicate that the nanosprings possess the capability to effectively reinforce the mechanical property, meanwhile significantly cutting the HL of elastomeric polymer materials, which indicates that carbon nanosprings as a kind of reinforcing filler could be a good candidate to prominently reduce the fuel consumption in the field of automobile tire industry.

\section{Methodology}

\subsection{Simulations}

Polymer chains are modeled through a bead-spring model, which is the same as our previous simulation work, ${ }^{[29]}$ and the pioneering polymer simulation work carried out by Kremer and Grest. ${ }^{[30]}$ In our simulations, the idealized elastomeric polymer chain consists of 30 beads with the mass $m$ and diameter $\sigma$ of each bead equal to unit. The total number of simulated polymer beads is 6000. Although these chains are rather short, they already show the static and dynamic behavior characteristic of long chains. Each bond in this model would correspond to $n=3-6$ covalent bonds along the backbone of a realistic chemical chain when mapping the coarse-grained model to a real polymer. The truncated and shifted Lennard-Jones (LJ) potential for non-bonded interactions between all beads is below:

$U(r)=\left\{\begin{array}{cl}4 \varepsilon\left[\left(\frac{\sigma}{r}\right)^{12}-\left(\frac{\sigma}{r}\right)^{6}\right]+C, & r<2.5 \sigma \\ 0, & r \geq 2.5 \sigma\end{array}\right.$

where the LJ interaction is cut off at the distance $r=2.5 \sigma$ and $C$ is a constant, which guarantees that the potential energy is continuous at the cutoff distance. $r$ is the separation distance between two polymer beads. $\sigma$ defines the length scale and $\varepsilon$ is the temperature scale of our model. Because it is not our aim to study a specific polymer, we use the LJ units where $\varepsilon$ and $\sigma$ are set to unit. This means that all calculated quantities are dimensionless. The interaction between the adjacent bonded monomers is modeled by a stiff finite extensible nonlinear elastic (FENE) potential:

$U_{\mathrm{FENE}}=-0.5 k R_{0}^{2} \ln \left[1-\left(\frac{r}{R_{0}}\right)^{2}\right]$

where $k=30 \frac{\varepsilon}{\sigma^{2}}$ and $R_{0}=1.5 \sigma$, guaranteeing a certain stiffness of the bonds while avoiding high-frequency modes and chain crossing. $r$ is the separation distance between two connected polymer beads. The nanospring is simply modeled as curved rod, as shown in Figure 1b. The neighboring beads are still controlled by the FENE potential, as shown in Equation (2). Meanwhile, the shape of the nanospring is governed by the following harmonic potential:

$U_{\text {angle }}=K\left(\theta-\theta_{0}\right)^{2}$

where $K / \varepsilon$ is varied to simulate different elastic behaviors of nanosprings, and $\theta_{0}$ is equal to $60^{\circ}$ or $180^{\circ}$ to keep the shape of the nanospring. It is noted that every nanospring is composed 
(a)
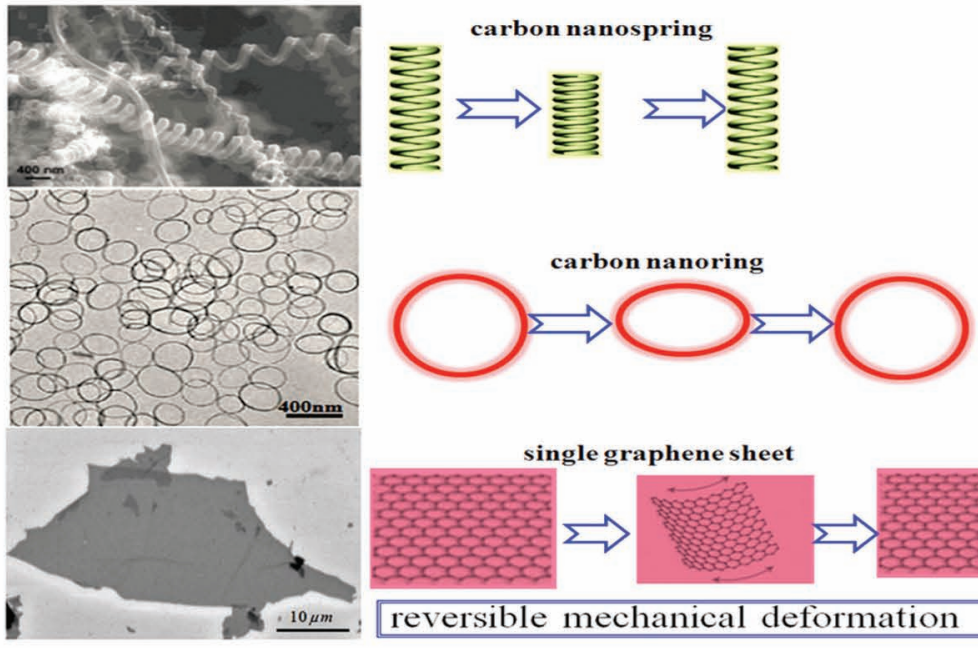

(b)
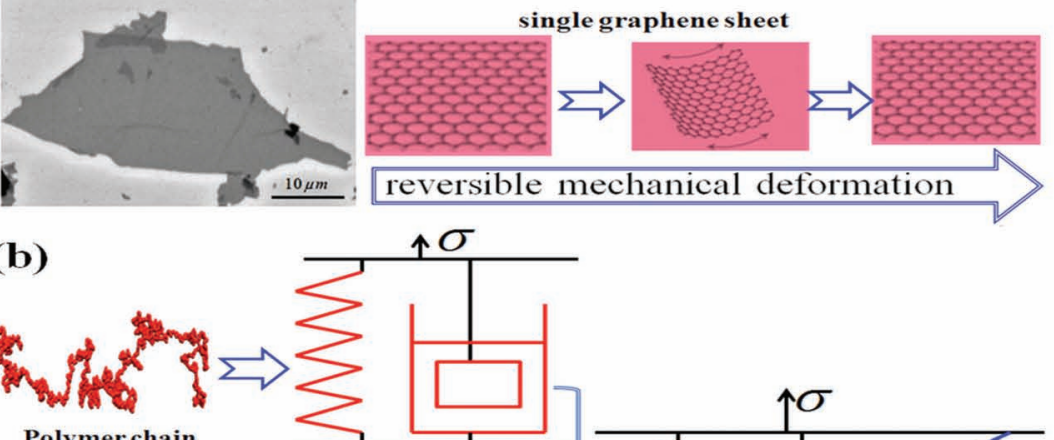

single graphene sheet

Polymerchain
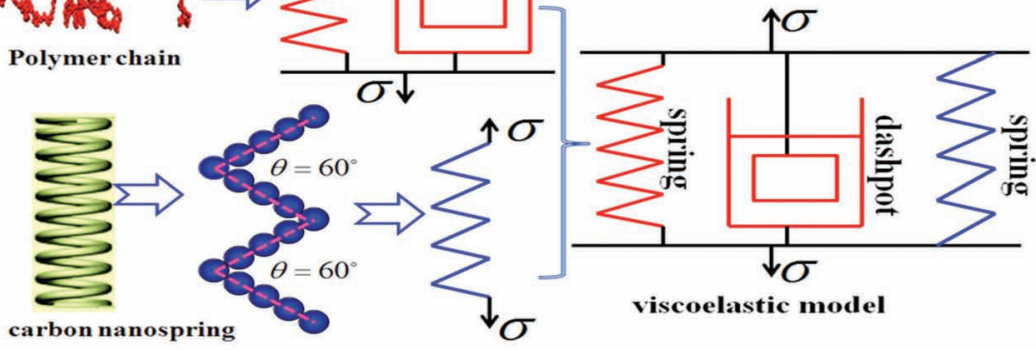

(c)

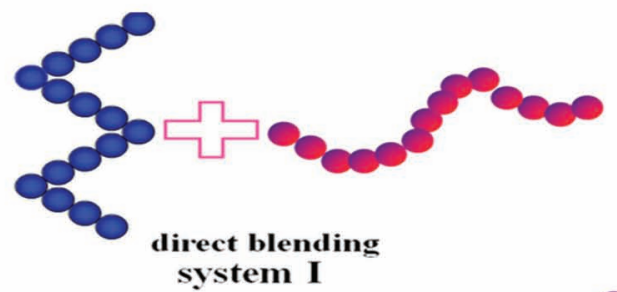

system I

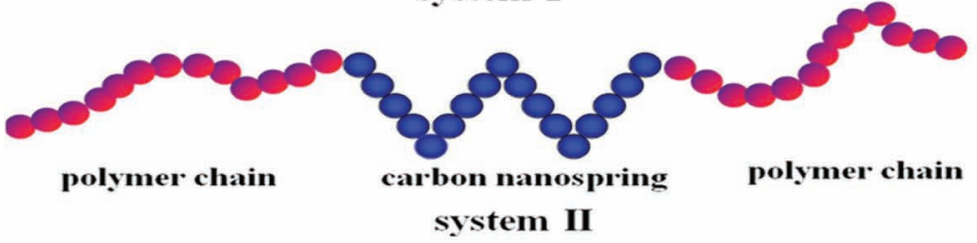

Figure 1. Schematics of the reversible mechanical deformation of a carbon nanospring, nanocoil, and graphene; the viscoelastic model of nanospring filled polymer chains; and two typical simulated systems. a) The SEM image of a carbon nanospring. Reproduced with permission. ${ }^{[13]}$ Copyright 2003, American Chemical Society. The TEM image of CNT rings. Reproduced with permission. ${ }^{14]}$ Copyright 2011, American Chemical Society. The SEM image of a single graphene sheet. Reproduced with permission. ${ }^{[40]}$ Copyright 2008, Nature Publishing Group. The schematics representing their reversible mechanical deformation. b) The viscoelastic model of carbon nanosprings filled elastomeric polymer systems, making up of the parallel arrangement of two springs and a dashpot. Note that in our simulation the carbon nanospring is represented by a simplified coarse-grained spring with the curved angle $\theta=60^{\circ}$. c) Two systems filled with nanosprings are constructed: system I is obtained by directly mixing nanosprings with polymer chains and system II is the self-assembly of tri-block chain-nanospringchain structure. of 29 beads, with its mass $m$ and diameter $\sigma$ also equal to unit. We have set the number of the nanosprings equal to 100 in every simulation box, corresponding to its volume fraction $\varphi=14 \%$. The interaction between polymer and nanosprings $U_{\mathrm{np}}$, and between nanosprings and nanosprings $U_{\mathrm{nn}}$ is shown below:

$$
U_{n p}(r)=\left\{\begin{array}{cc}
4 \varepsilon_{n p}\left[\left(\frac{\sigma}{r}\right)^{12}-\left(\frac{\sigma}{r}\right)^{6}+C_{1}\right], & r>2.5 \sigma \\
\infty, & r \leq 2.5 \sigma
\end{array}\right.
$$

$U_{n n}(r)=\left\{\begin{array}{cc}4 \varepsilon_{n n}\left[\left(\frac{\sigma}{r}\right)^{12}-\left(\frac{\sigma}{r}\right)^{6}+C_{2}\right], & r>2^{1 / 6} \sigma \\ \infty, & r \leq 2^{1 / 6} \sigma\end{array}\right.$

where $C_{1}$ and $C_{2}$ are constants, which assure that the potential energy is continuous at the cutoff distance. $\varepsilon_{\mathrm{nn}}$ and $\varepsilon_{\mathrm{np}}$ are both set to be 1.0. Notedly, for good dispersion the interaction between the nanosprings is purely repulsive. For good comparison, the parameters of interaction potential energy between polymer-polymer, polymer-nanosprings and nanosprings-nanosprings are listed in Table 1.

In our MD simulation, during equilibration the NPT ensemble is adopted, where the temperature is fixed at $T^{*}=1.0$, which is far above the glass transition temperature $\left(T^{*}=0.41\right)$ of polymer chains, ${ }^{[31]}$ and the pressure is set to be $P^{*}=0.03$ by using the NoseHoover thermostat and barostat. ${ }^{[32]}$ Periodic boundary conditions are employed in all three directions during the simulation. The velocity-Verlet algorithm is used to integrate the equations of motion, with a timestep $\delta t=0.001$, where the time is reduced by LJ time $(\tau)$. Before cross-linking, for system I, the longest relaxation of polymer chains can be characterized by the end-to-end vector autocorrelation function $\langle u(t) \cdot u(0)\rangle$, with $u(t)$ being the unit vector along the end-to-end vector of a chain. And its decay rate can reflect how fast polymer chain forgets its initial configuration, as shown in Figure S1a (Supporting Information). After this long equilibration $\left(1.5 \times 10^{7} \mathrm{MD}\right.$ steps $)$, the change of the potential energy of both systems are plotted in Figure S1b (Supporting Information). Meanwhile, for system I, the change of the mean square end-to-end distance $R^{2}$ eed and radius of gyration $R^{2}$ are presented in Figure S1c (Supporting Information). Obviously, they exhibit small fluctuations. After this, permanent cross-links are imposed between polymer chains by randomly selecting pairs of beads within a 
Table 1. Parameters of interaction potential energy between polymer-polymer, polymer-nanosprings and nanosprings-nanosprings.

\begin{tabular}{lcccc}
\hline polymer-polymer & & $\begin{array}{c}\text { polymer- } \\
\text { nanosprings }\end{array}$ & nanosprings-nanosprings \\
\hline non-boned & boned & L) potential & non-bonded & bonded \\
LJ potential & FENE potential & & L) potential & $\begin{array}{c}\text { harmonic } \\
\text { potential }\end{array}$ \\
$\varepsilon=1.0$ & $k=30 \frac{\varepsilon}{\sigma^{2}}$ & $\varepsilon_{\text {np }}=1.0$ & $\varepsilon_{\text {nn }}=1.0$ & $K / \varepsilon=0.05,50$, \\
$\sigma=1.0$ & $R_{0}=1.5 \sigma$ & $\sigma=1.0$ & $\sigma=1.0$ & $500 \theta_{0}=60^{\circ}$ or \\
$r_{\text {cutoff }}=2.5 \sigma$ & & $r_{\text {cutoff }}=2.5 \sigma$ & $r_{\text {cutoff }}=1.12 \sigma$ & $180^{\circ}$ \\
\hline
\end{tabular}

distance of $1.25 \sigma$ of each other, and tethering them together by means of the interaction potential (Equation (2)). Systems I and II have the same cross-linking density with the number of the cross-linked bonds equal to 1200 . During further equilibration $\left(2 \times 10^{6}\right.$ MD runs) with NPT ensemble, we record the change of the potential energy and number density of both systems in the last $1 \times 10^{6} \mathrm{MD}$ runs, as shown in Figure S2a,b (Supporting Information). These thermodynamic values approach to be nearly unchanged. Based on these results, we believe that our simulated systems have been fully and properly equilibrated.

Because the elastomer network is typically incompressible, with its Poisson's ratio $\mu \approx 0.5$, its volume almost does not change when being deformed. For uniaxial deformation, when the network is stretched in one direction, the other two directions of the network will simultaneously adjust to keep the volume constant. ${ }^{[41]}$ Hence, the pure and nanosprings filled elastomer network systems are deformed in one direction and meanwhile, in the other two directions, they are relaxed to keep the volume of the simulation box unchanged, which in fact is also consistent with the simulation method used by Curro et al. ${ }^{[35-37]}$ We set the tensile and recovery strain rate $\left.\dot{\varepsilon}=\left(L_{z}(t)-L_{z}\right) / L_{z}=0.0327\right) \tau$ (i.e., in the $z$-direction), which is the same as the simulation work from Gao et al. ${ }^{[33,34]}$ The average stress $\sigma$ in the tensile direction (i.e., $z$-direction) is obtained from the deviatoric part of the stress tensor $\sigma=(1+\mu)\left(-P_{z z}+\right.$ $P) \approx 3\left(-P_{z z}+P\right) / 2$, where $P=\sum_{i} P_{i i} / 3$ is the hydrostatic pressure, which is also followed from Curro et al. ${ }^{[35-37]}$ All MD simulations are carried out through the large scale atomic/molecular massively parallel simulator (LAMMPS), which was developed by Sandia National Laboratories. ${ }^{[38]}$ More detailed simulation techniques can be found in our previous published work. ${ }^{[29,39]}$

\subsection{Experimental Section}

To examine the microscopic deformation of carbon nanosprings in the elastomer matrix, first we mix natural rubber with carbon nanosprings on a two-roll mill, and then it is vulcanized at nearly $150^{\circ}$. After this, the dumbbell shaped sample is used to perform the uniaxial tension. During the tension and recovery process, the optical microscope is used to characterize the microscopic deformation of carbon nanosprings. In order for clear observation through optical microscope, we have used carbon nanosprings with large diameter and aspect ratio, as shown in Figure S3 (Supporting Information) observed by TEM. The experimental result is shown in Figure S4 (Supporting Information). Obviously, the carbon nanosprings gradually straighten when the strain increases from $100 \%$ to $300 \%$. Furthermore, during the recovery process, the straightened carbon nanosprings become curved again, indicating that the carbon nanosprings have the ability to undergo flexible and recoverable mechanical deformation. This direct experimental observation can serve as a basis to use carbon nanosprings to adjust the viscoelasticity of elastomers.

\section{Results and Discussion}

In Figure 1a the microscopic morphology of carbon nanosprings, nanorings and graphene obtained from the literature $\mathrm{e}^{[13,14,40]}$ are shown, and their corresponding reversible mechanical deformation are also displayed. The viscoelastic model of nanosprings filled elastomeric polymer chains is also shown in Figure 1b, making up of the parallel arrangement of two springs and a dashpot. Note that in our simulation the carbon nanosprings are represented by a simplified coarsegrained spring with the elastic deformation angle $\theta=60^{\circ}$, which, however, have captured the fundamental physical characteristics of carbon nanosprings, such as its high flexibility and reversible elastic deformation. In our work we construct and compare two typical systems I and II, which are displayed in Figure 1c. System I is obtained by directly mixing nanosprings

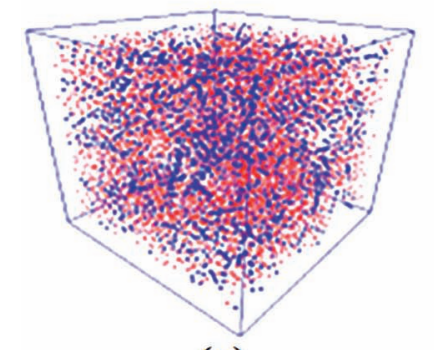

(a)

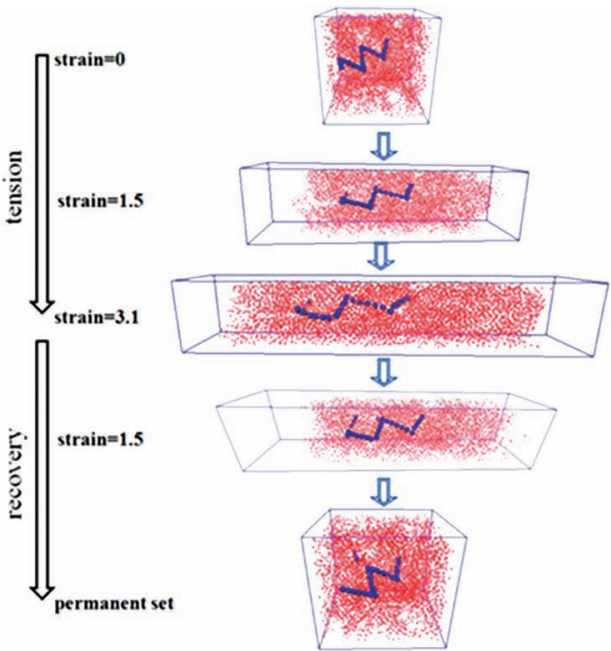

(b)

Figure 2. Snapshots of nanosprings filled cross-linked polymer system (system I). a) The homogeneous dispersion of the nanosprings in polymer chains and b) the microscopic deformation of the nanospring during tension-recovery process. Note that the blue dots represent the nanosprings, and the red dots denote the polymer chains. For clarity only one nanospring is shown. The volume fraction of the nanosprings is $\varphi=14 \%$ and the dimensionless spring constant is $K^{*}=500$ with $K^{*}=K / \varepsilon$. 
with polymer chains, and system II is the self-assembly of the triblock chain-nanospring-chain structure. It is noted that for both systems the volume fraction of the nanosprings is nearly $\varphi=14 \%$.

To examine the nanospring effect on the hysteresis loss, we focus our attention on the permanent set of the tensionrecovery process. Theoretically, a large permanent set always means great slippage and internal friction between elastomeric macromolecule chains, consequently resulting in more hysteresis loss. ${ }^{[41]}$ Moreover, we also calculate the hysteresis loss from the tension-recovery stress-strain curve in one cycle in order for quantitative comparison. Firstly we characterize system I, namely directly mixing nanosprings with polymer chains, after which polymer chains are further cross-linked, and interfacial chemical links are also produced to enhance interfacial interaction between nanosprings and polymer chains. Since the interactions between polymer chains and polymer chains, nanosprings and polymer chains are the same, we obtain a relatively homogeneous dispersion of nanosprings in polymer chains, as shown in Figure 2a. The spring constant of the nanosprings $K^{*}$ is set to 500, possessing enough elastic behavior. Snapshots shown in Figure $2 \mathrm{~b}$ displays the microscopic deformation of the nanosprings during the tension-recovery process, clearly showing the reversible elastic deformation of the nanosprings.

Without interfacial chemical links, the tension-recovery stress-strain curves in the $x, y$, and $z$ directions are presented in Figure S5 (Supporting Information). For good comparison, we also add the case for the pure system with only cross-linked polymer chains with the same cross-linking density. Obviously, since the nanosprings are nearly homogeneously dispersed in the polymer chains, the stress-strain tension-recovery curves are almost the same in the $x, y$, and $z$ directions (for clarity, the result in the $z$-direction is not shown). Moreover, in comparison with the pure system, the incorporation of the nanosprings decrease the permanent set to some extent.

The interfacial binding strength always has great influence on the static and dynamic mechanical properties of elastomer nanocomposites and in practical situations some interfacial coupling agents are always used to strengthen the interfacial interaction. ${ }^{[42]}$ Therefore, we also investigate the effect of the interfacial links on the stress-strain curves and permanent set, as shown in Figure 3a. Obviously, with the increase of the number of the interfacial links ranging from $M=0,200,400$, 600 , and 800 , corresponding to the interfacial chemical coupling density (defined as the ratio of the number of the interfacial links $M$ to the total box volume) $\rho^{*}=0,0.019,0.038,0.057$, and 0.075 , the stress-strain behaviors are enhanced, exhibiting better mechanical reinforcement performance. For instance, in comparison with the pure cross-linked system, the stress at the strain $\varepsilon=300 \%$ for the system with the interfacial chemical cross-linking density $\rho^{*}=0.075$ is almost three times than that of the pure system. Meanwhile, it is observed that the permanent set is also gradually decreasing, which can be attributed to the fact that enhanced polymer-filler interaction leads to better synergistic motion of the nanosprings and the macromolecule chains during the tension-recovery process. Additionally, in Figure $3 \mathrm{~b}$ we also show the change of the elastic energy during the tension-recovery process for different interfacial chemical cross-linking density $\rho^{*}$. Reasonably, with the increase of $\rho^{*}$,
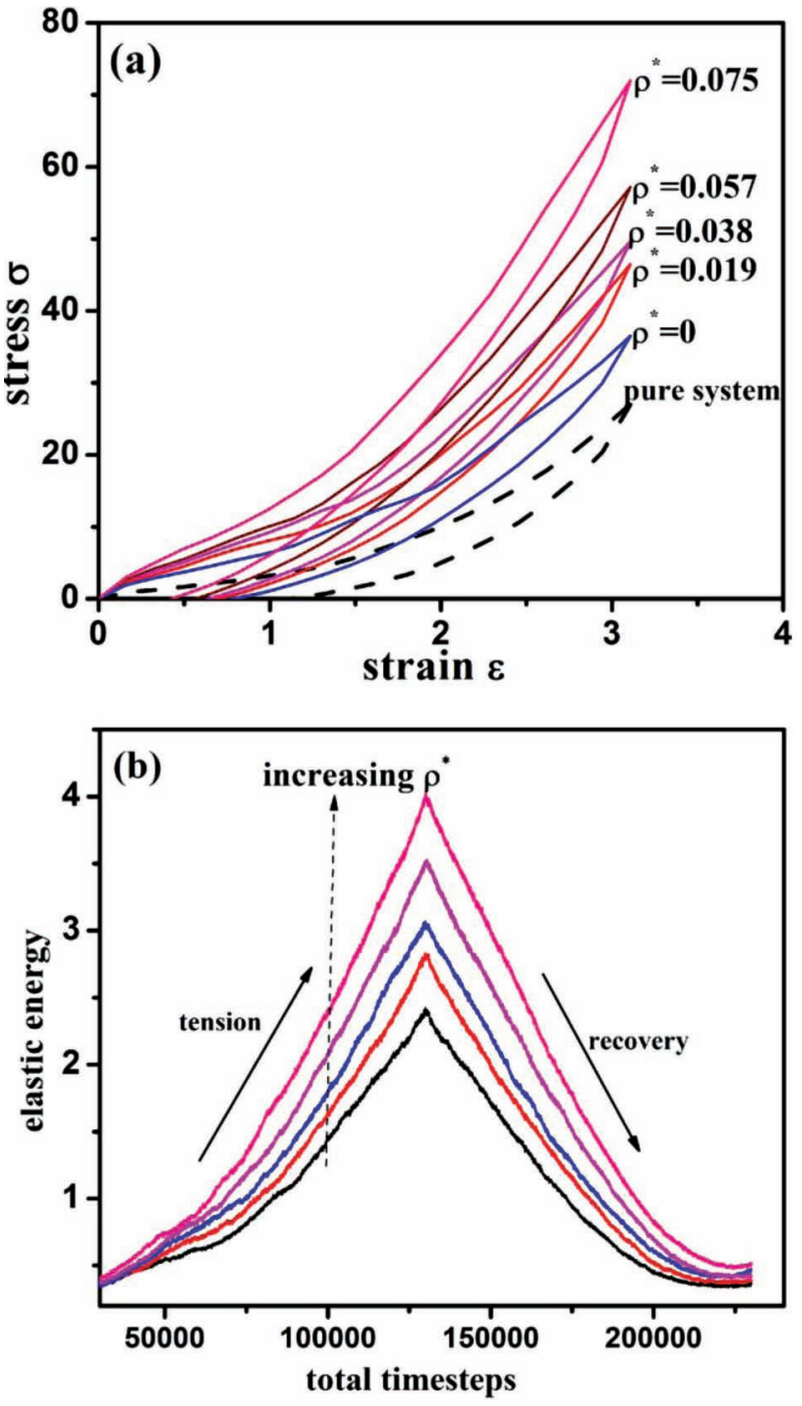

Figure 3. For nanosprings filled cross-linked polymer system (system I): a) comparison of the tension-recovery curves for different interfacial chemical coupling density, denoted by $\rho^{*}$ and b) comparison of the elastic energy of nanosprings for different interfacial chemical coupling density $\rho^{*}=0,0.019,0.038,0.057$ and 0.075 during the tension-recovery process. The volume fraction of the nanosprings is $\varphi=14 \%$ and the spring constant is $K^{*}=500$.

more stress can be transferred to the nanosprings, and greater increase of the elastic energy is observed during the tension process. However, the entire change of the elastic energy indicates that the nanosprings can almost recover to their initial state during the recovery process.

In addition to the effect of the interfacial interaction, we also characterize the effect of the nanosprings by changing the spring constant $K^{*}$. For cross-linked system with the interfacial links $M=800$ and $M=0$, we purely change the spring constant $K^{*}$, and the result is shown in Figure 4a,b. Comparing between the spring constant $K^{*}=500$ and $K^{*}=0$ (the nanosprings have no elastic behavior with $K^{*}=0$ ), the stress-strain behavior and 

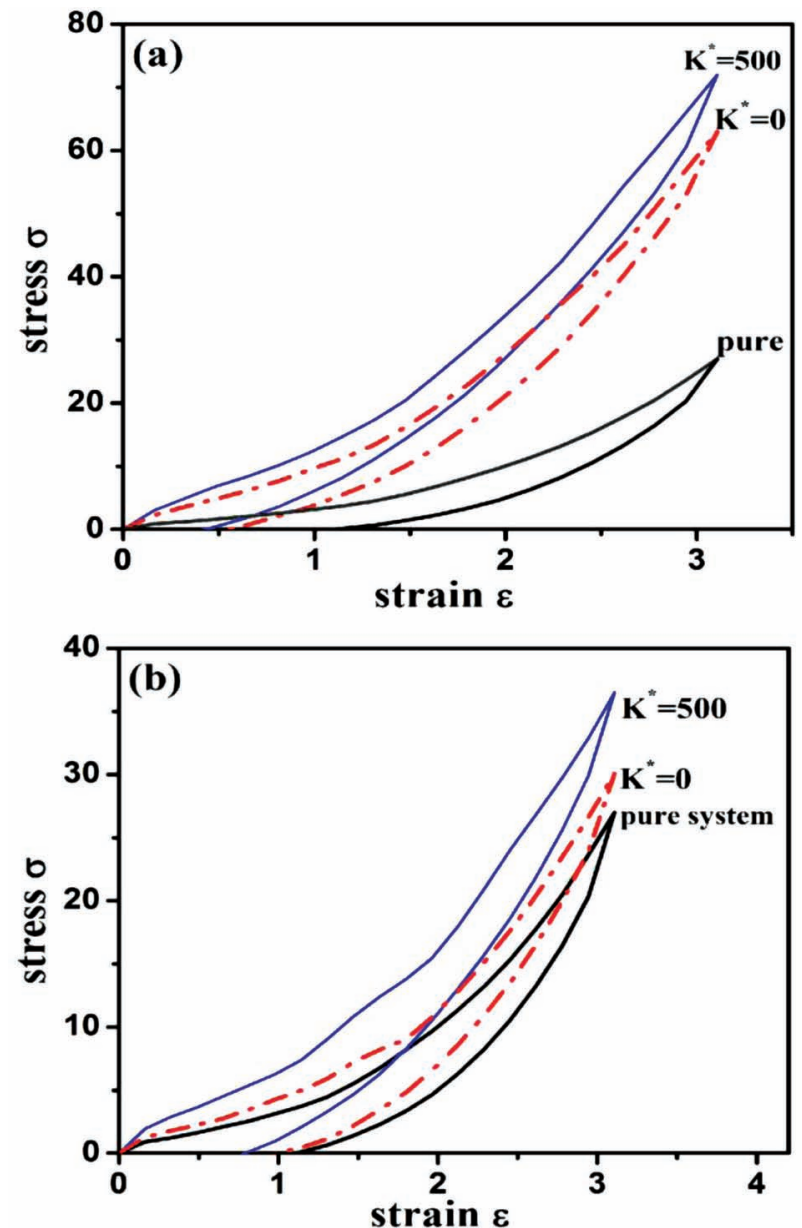

Figure 4. For nanosprings filled cross-linked polymer system (system I), comparison of the tension-recovery curves a) with the interfacial chemical coupling density $\rho^{*}=0.075$ for two special spring constants $K^{*}=500$ and $K^{*}=0$ and $b$ ) without interfacial chemical coupling for two special spring constants $K^{*}=500$ and $K^{*}=0$. The volume fraction of the nanosprings is $\varphi=14 \%$.

the permanent set change moderately for both systems with or without interfacial chemical links. Namely, the results reveal that the permanent set decreases with the increase of the spring constant, since greater spring constant will store more recoverable elastic energy, leading to less permanent set during the tension-recovery deformation.

Generally, for system I, in comparison with the pure system, the incorporation of the nanosprings can strengthen the mechanical property of the elastomeric polymer network and simultaneously decrease the permanent set during the tension-recovery process. These effects become more prominent by increasing the interfacial chemical links between polymer chains and nanosprings. It should be pointed out that the spring constant $K^{*}$ is also an important factor in controlling the permanent set. This remarkable simulated result just validates our preliminary assumption that the introduction of the nanosprings could reduce the hysteresis of the elastomer matrix.

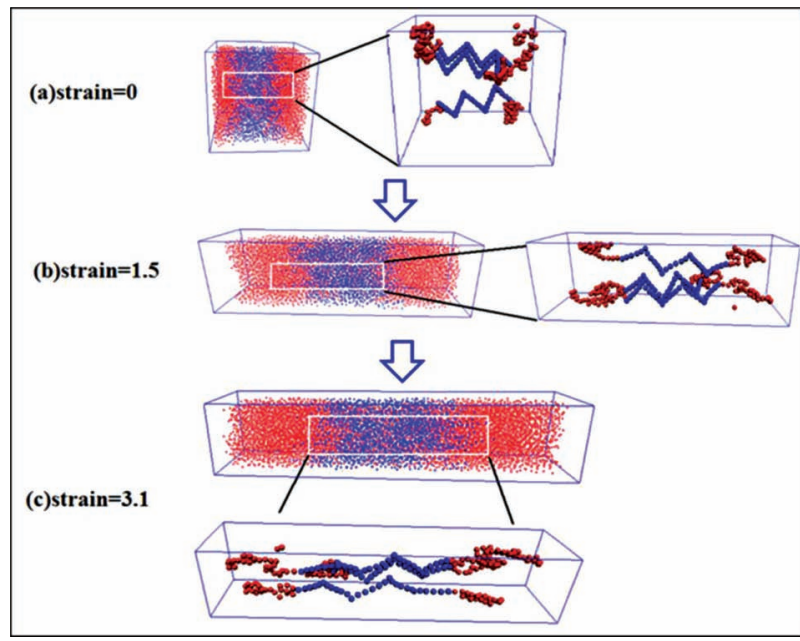

Figure 5. For nanosprings filled cross-linked polymer system (system II) with the elastic constant $K^{*}=500$, snapshots of the microscopic deformation during the tensile deformation along the $y$ direction at three typical strains: a) strain $=0$, b) strain $=1.5$, and c) strain $=3.1$. The volume fraction of the nanosprings is $\varphi=14 \%$.

In the following part we extend our efforts to study system II. The equilibrated structure through the self-assembly process is shown in Figure 5, which forms a lamellae structure, with the orientation of the nanosprings along the $y$-direction. Then the cross-linking bonds are further produced between polymer chains with the number equal to 1200 , corresponding to the cross-linking density equal to 0.113 , which is the same as system I. The snapshots in Figure 5 reveal the microscopic deformation of the nanosprings only for the tension process without the recovery process along the $y$-direction, namely the orientation direction of the nanosprings. Obviously, the nanosprings gradually exhibit greater elastic deformation during the tension process.

For system II, we purely change the spring constant of the nanosprings $K^{*}$ to examine its effect on the viscoelastic property, as shown in Figure $\mathbf{6}$. The tension-recovery process is carried out in the $\gamma$-direction. Significantly, the permanent set for the spring constant $K^{*}=500$ becomes only 0.25 , which is much smaller than that of system I with $K^{*}=500$ (see Figure $4 \mathrm{~b}$ ), and interestingly for the spring constant $K^{*}=0.05$, the permanent set exhibits an abrupt change to be 1.0 , which is nearly the same as the pure system. To further examine the microscopic deformation mechanism, we compare the permanent set of the tension-recovery process in the $x, y$, and $z$ directions with the spring constant $K^{*}=500$ and $K^{*}=0.05$, as shown in Figure S6a and Figure S7a (Supporting Information). We also show the corresponding change of the elastic energy in the $x, y$, and $z$ directions in Figure S6b and Figure S7b (Supporting Information). From these results, it is observed that for the spring constant $K^{*}=500$, the permanent set in the $y$ direction is prominently smaller than those in both $x$ and $z$ directions, and correspondingly the elastic energy in the $\gamma$ direction is also more significant than those in $x$ and $z$ directions during the tensionrecovery process. These results are attributed to the anisotropic structure formed by the self-assembly, with the nanosprings 


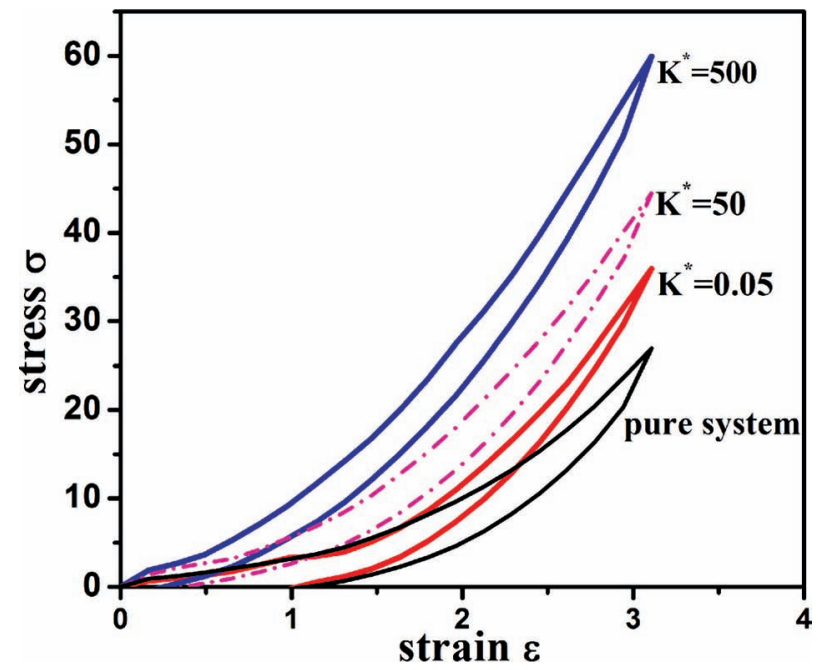

Figure 6. For the nanospring filled cross-linked polymer system (system II), comparison of the tension-recovery curves between different spring constants $K^{*}$ of the nanosprings. For good comparison the case for pure system is also added. The volume fraction of the nanosprings $\varphi=14 \%$ and the dimensionless spring constant $K^{*}=K / \varepsilon$.

orientating in the $y$ direction. While for much smaller spring constant $K^{k}=0.05$, which leads to the loss of the capability of the recoverable elastic deformation for nanosprings, the permanent set in the $x, y$, and $z$ directions becomes nearly the same in this case, evidenced by Figure S7a (Supporting Information), and the change of the elastic energy is small and nearly the same in the $x, y$, and $z$ directions, as shown in Figure S7b (Supporting Information). Hence, the effect for the nanosprings to adjust the viscoelasticity of elastomer matrix is more prominent through the self-assembly process, in comparison with the homogeneous dispersion of the nanosprings by direct mixing. However, for system I and II the spring constant $K^{*}$ plays a very crucial role.

In order to further examine the quantitative effect of the nanosprings on the viscoelasticity, we can directly calculate the hysteresis loss, namely the ratio between the loss energy to the initial storage energy in one tension-recovery cycle. ${ }^{[41]}$ For system I, we focus on the effect of the interfacial chemical coupling density $\rho^{*}$ on the hysteresis loss, as shown in Figure 7a. The hysteresis loss decreases with the increase of $\rho^{*}$. Furthermore, for system II, the effect of the spring constant $K^{*}$ on the hysteresis loss is presented in Figure 7b. For better comparison, the case with $K^{*}=500$ and $\rho^{*}=0.075$ of system I is also added. It is evident that with the increase of the spring constant $K^{*}$, the hysteresis loss decreases significantly, for instance, the hysteresis loss becomes $18 \%$ for system II with $K^{*}=500$, compared to the hysteresis loss equal to $40 \%$ for the pure cross-linked elastomer system, and the hysteresis loss for system II with $K^{*}=$ 500 is much lower than all cases of system I. These quantitative comparisons are consistent with the change of the permanent set discussed above. In comparison with the pure system, it is convincing that the incorporated nanosprings could reinforce the elastomeric polymer materials while meanwhile decrease the hysteresis loss.
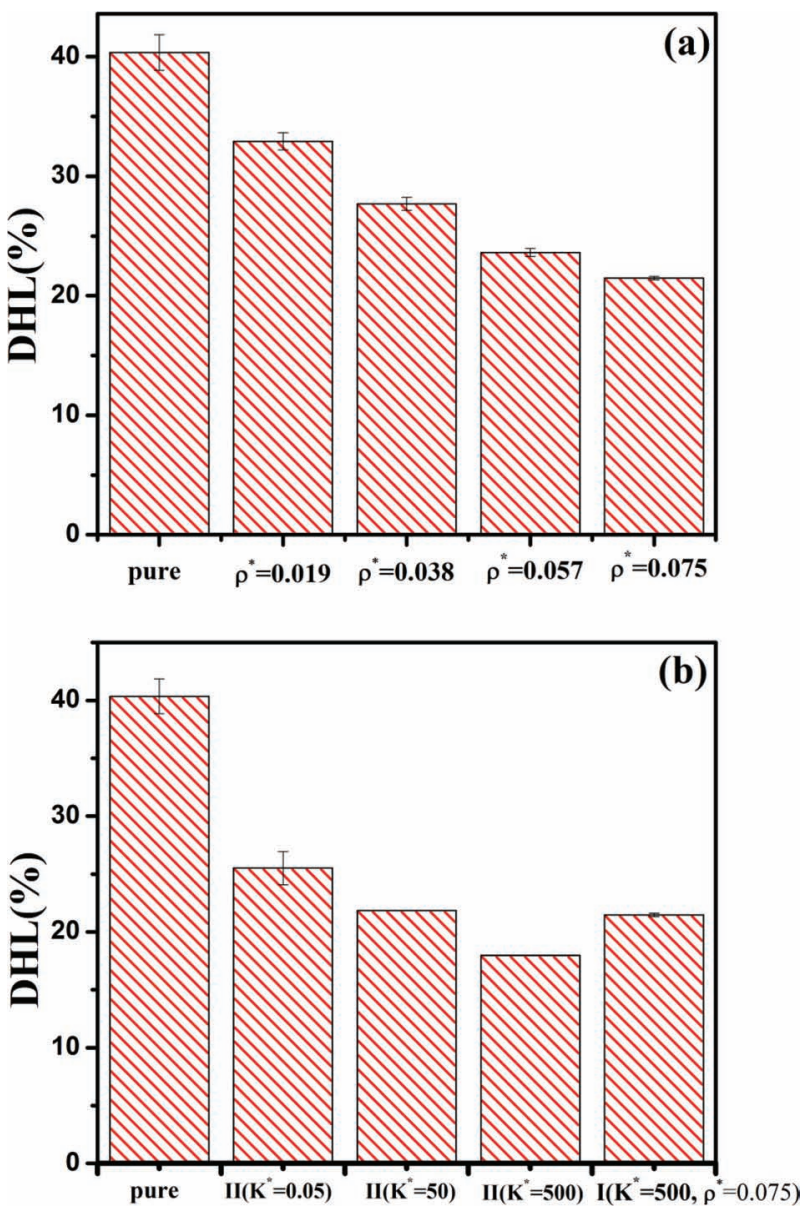

Figure 7. Comparison of the hysteresis loss (HL). a) For nanosprings filled cross-linked polymer system (system I), the effect of the interfacial chemical coupling density $\rho *$ between nanosprings and polymer chains on the hysteresis loss (HL). b) For nanospring filled cross-linked polymer system (system II), the effect of the spring constant $K^{*}$ of nanosprings on the $\mathrm{HL}$, for better comparison the case with $K^{*}=500$ and $\rho^{*}=0.075$ of system $I$ is also added. The volume fraction of the nanosprings is $\varphi=$ $14 \%$. Note that the dimensionless spring constant is denoted by $K^{*}$ and $\rho *$ represents the dimensionless interfacial chemical coupling density.

\section{Conclusions}

To further exploit the practical application of carbon nanostructured materials in the field of nanoscience and nanotechnology, we draw attention to the use of carbon nanosprings to adjust the viscoelasticity of elastomeric polymer materials. We mainly focus on two systems: system I is obtained by directly blending nanosprings with polymer chains, while system II is the selfassembly of the tri-block chain-nanospring-chain structure. The simulated results indicate that in addition to possessing great reinforcing capability, the nanosprings decrease the permanent set for both systems in comparison with the pure case and considerably reduce the hysteresis loss (HL) and the resulting rolling resistance, which is attributed to the reversible storage and release of elastic energy of nanosprings. This finding is of great significance for reducing fuel consumption in the automobile tire industry. Moreover, it seems that the adjustment 
effect is more prominent for system II than system I because of the anisotropic orientation and alignment of the nanosprings through self-assembly. Importantly, the spring constant $K^{*}$ plays a crucial role for both system I and II. Meanwhile, the introduction of the interfacial chemical links between nanosprings and polymer chains will further decrease the permanent set, which fundamentally results from the synergistic motion between the nanosprings and the surrounding polymer chains. On the basis of the present work, it is inferred that with good dispersion and interfacial interaction, some other carbon nanostructured materials with good flexibility and reversible elastic deformation, such as carbon nanocoils, ${ }^{[13]}$ nanorings, ${ }^{[14,43]}$ or thin graphene sheets ${ }^{[15,16]}$ could also be introduced to both enhance the mechanical properties and significantly decrease the HL of elastomeric polymer materials, which likely provides a promising opportunity to achieve the large-scale industrial application of carbon nanostructured materials.

\section{Supporting Information}

Supporting Information is available from the Wiley Online Library or from the author.

\section{Acknowledgements}

This work was supported by the National Basic Research Program of China (973 Program)(2011CB932603), Foundation for Innovative Research Groups of the NSF of China (51221002), Outstanding Young Scientists Foundation of NSF of China (50725310), and National High Technology Research and Development Program of China (863 Program) (2009AA03Z338).

Received: May 29, 2012

Revised: September 2, 2012 Published online: October 9, 2012

[1] A. S. Arico, P. Bruce, B. Scrosati, J. M. Tarascon, W. Van Schalkwijk, Nat. Mater. 2005, 4, 366.

[2] A. Modi, N. Koratkar, E. Lass, B. Q. Wei, P. M. Ajayan, Nature 2003, 424, 171.

[3] P. W. Barone, S. Baik, D. A. Heller, M. S. Strano, Nat. Mater. 2005, $4,86$.

[4] S. M. Jung, J. Hahn, H. Y. Jung, J. S. Suh, Nano Lett. 2006, 6, 1569.

[5] B. Y. Lee, K. Heo, J. H. Bak, S. U. Cho, S. Moon, Y. D. Park, S. Hong, Nano Lett. 2008, 8, 4483.

[6] M. Cadek, J. N. Coleman, K. P. Ryan, V. Nicolosi, G. Bister, A. Fonseca, J. B. Nagy, K. Szostak, F. Beguin, W. J. Blau, Nano Lett. 2004, 4, 353.

[7] B. S. Shim, J. Zhu, E. Jan, K. Critchley, S. S. Ho, P. Podsiadlo, K. Sun, N. A. Kotov, ACS Nano 2009, 3, 1711.

[8] M. Moniruzzaman, K. I. Winey, Macromolecules 2006, 39, 5194.
[9] T. J. Simmons, J. Bult, D. P. Hashim, R. J. Linhardt, P. M. Ajayan, ACS Nano 2009, 3, 865.

[10] M. A. Poggi, J. S. Boyles, L. A. Bottomley, Nano Lett. 2004, 4, 1009.

[11] A. F. da Fonseca, D. S. Galvao, Phys. Rev. Lett. 2004, 92, 175502.

[12] A. F. da Fonseca, C. P. Malta, D. S. Galvao, Nanotechnology 2006, $17,5620$.

[13] X. Q. Chen, S. L. Zhang, D. A. Dikin, W. Q. Ding, R. S. Ruoff, L. J. Pan, Y. Nakayama, Nano Lett. 2003, 3, 1299.

[14] L. Y. Chen, H. Wang, J. Xu, X. S. Shen, L. Yao, L. F. Zhu, Z. Y. Zeng H. Zhang, H. Y. Chen, J. Am. Chem. Soc. 2011, 133, 9654.

[15] C. Gomez-Navarro, M. Burghard, K. Kern, Nano Lett. 2008, 8, 2045.

[16] F. Scarpa, S. Adhikari, A. S. Phani, Nanotechnology 2009, 20, 065709.

[17] M. Xu, D. N. Futaba, M. Yumura, K. Hata, Adv. Mater. 2011, 23, 3686.

[18] M. Xu, D. N. Futaba, M. Yumura, K. Hata, Nano Lett. 2011, 11, 3279.

[19] M. Xu, D. N. Futaba, T. Yamada, M. Yumura, K. Hata, Science 2010, 330,1364

[20] Y. Gogotsi, Science 2010, 330, 1332.

[21] L. M. Dai, Angew. Chem. Int. Ed. 2011, 50, 4744.

[22] S. lijima, C. Brabec, A. Maiti, J. Bernholc, J. Chem. Phys. 1996, 104, 2089.

[23] M. R. Falvo, G. J. Clary, R. M. Taylor, V. Chi, F. P. Brooks, S. Washburn, R. Superfine, Nature 1997, 389, 582.

[24] L. Q. Zhang, Y. P. Wu, Y. Q. Wang, Y. Z. Wang, China Synthetic Rubber Industry 2000, 23, 71 .

[25] G. R. Hamed, Rubber Chem. Technol. 2000, 73, 524.

[26] J. D. Ferry, Viscoelastic Properties of Polymers, John Wiley and Sons, New York 1980.

[27] S. Merabia, P. Sotta, D. R. Long, Macromolecules 2008, 41, 8252.

[28] M. Daniel, C. Janin, Actual. Chim, 2010, 340, 8.

[29] J. Liu, D. P. Cao, L. Q. Zhang, W. C. Wang, Macromolecules 2009, 42, 2831.

[30] K. Kremer, G. S. Grest, J. Chem. Phys. 1990, 92, 5057.

[31] K. Binder, J. Baschnagel, W. Paul, Prog. Polym. Sci. 2003, 28, 115.

[32] M. P. Allen, D. J. Tildesley, Computer Simulation of Liquids, Oxford University Press, New York 1987.

[33] J. P. Gao, J. H. Weiner, Science 1994, 266, 748.

[34] J. Gao, J. H. Weiner, Macromolecules 1991, 24, 1519.

[35] D. R. Rottach, J. G. Curro, G. S. Grest, A. P. Thompson, Macromolecules 2004, 37, 5468.

[36] D. R. Rottach, J. G. Curro, J. Budzien, G. S. Grest, C. Svaneborg, R. Everaers, Macromolecules 2006, 39, 5521.

[37] D. R. Rottach, J. G. Curro, J. Budzien, G. S. Grest, C. Svaneborg, R. Everaers, Macromolecules 2007, 40, 131.

[38] S. Plimpton, J. Comput. Phys. 1995, 117, 1.

[39] J. Liu, Y. G. Gao, D. P. Cao, L. Q. Zhang, Z. H. Guo, Langmuir 2011, $27,7926$.

[40] V. C. Tung, M. J. Allen, Y. Yang, R. B. Kaner, Nat. Nanotechnol. 2009, 4, 25

[41] M. Rubinstein, R. H. Colby, Polymer Physics, Oxford University Press, Oxford, UK 2003.

[42] C. G. Robertson, C. J. Lin, M. Rackaitis, C. M. Roland, Macromolecules 2008, 41, 2727.

[43] M. Zheng, C. H. Ke, Small 2010, 6, 1647. 